\title{
GEOMETRICAL PROPERTIES OF SKELETAL STRUCTURES OF RADIOLARIAN GENUS DIDYMOCYRTIS
}

\author{
TAKAshi Yoshino ${ }^{\bowtie, 1}$, Atsushi MatsuokA ${ }^{2}$ AND NaOKO Kishimoto ${ }^{3}$ \\ ${ }^{1}$ Department of Mechanical Engineering, Toyo University, 2100 Kujirai, Kawagoe 350-8585, Japan; \\ ${ }^{2}$ Department of Geology, Faculty of Science, Niigata University, Niigata 950-2181, Japan; ${ }^{3}$ Department of \\ Mechanical Engineering, Setsunan University, 17-8 Ikeda-Nakamachi, Neyagawa 572-8508, Japan \\ e-mail: tyoshino@toyo.jp, amatsuoka@geo.sc.niigata-u.ac.jp, kishimoto@mec.setsunan.ac.jp \\ (Received December 30, 2018; revised July 18, 2019; accepted July 18, 2019)
}

\begin{abstract}
This paper discusses the geometrical properties of a radiolarian skeletal structure, namely, that of genus Didymocyrtis. We characterized the evolution of skeletal structures and analyzed the structures using geometry. We defined two ratios in order to quantify the geometrical properties of Didymocyrtis and verified that the two ratios changed with their phylogenic evolution. We also used the 3D skeletal data of a specimen of species D. tetrathalamus, which were obtained through micro X-ray CT. The cortical shell obtained in the 3D data was projected onto a spherical surface, and we determined the centers of the pores. Our analysis revealed that the number of pores is approximately 200 and their distribution is not regular. We also determined that the column-like parts of the skeleton, which connect the inner and upper parts of the specimen, do not lie on a plane and their intervals are not equal.
\end{abstract}

Keywords: phylogenic evolution, skeletal structures of radiolarians, three-dimensional data.

\section{INTRODUCTION}

Radiolarians are a type of marine plankton having siliceous skeletons. Their fossils have been used as geological indexes for exploring the Earth's history. Studies of radiolarians are based on their skeletal structures because their soft parts dissolved before the earliest taphonomic processes, leaving only their hard parts. Therefore, extracting useful information from the skeletal structure is the main subject of such studies. Paleontologists do not use geometrical terms, so they describe the structures qualitatively. In order to discuss the structures quantitatively, the structures must be considered geometrically, but such approaches are not popular at present.

Recently, a method using micro X-ray CT to obtain three-dimensional (3D) skeletal data of radiolarians has been developed (Ishida et al., 2015; Wagner et al., 2015). However, the main object of the data acquisition by paleontologists is to observe the skeletal structures of the specimens in detail. This is because the target of research on radiolarians is to describe the structural similarities and differences from previously found species. Furthermore, no quantitative method for treating the data has yet been established.

There are three main difficulties in the quantitative analysis of radiolarian skeletons. Firstly, most of their shapes are not convex. Therefore, our knowledge of convex geometry cannot be applied directly to their shapes. Secondly, radiolarians are so small that they can only be observed using microscopes. Therefore, observation was always limited to confined angles until the introduction of the micro X-ray CT method (Matsuoka et al., 2012; Yoshino et al., 2015). Lastly, although the skeletal structures have many pores, a general method for analyzing the pores has not been developed. In the case of spherical skeletons, there have been some studies on the geometrical analysis of pore arrangements (Yoshino et al., 2014), but the methods of those studies do not extend to other shapes.

In the present paper, we discuss the skeletal structures of genus Didymocyrtis quantitatively by introducing a geometrical method. We intend to show through this paper that the proposed geometrical approach is effective for describing the skeletal structures of radiolarians. This paper consists of three parts: Firstly, we describe the genus of the radiolarians which we focused on. Secondly, we introduce some geometrical definitions in order to discuss the skeletal structures of some species which belong to that genus. Finally, we present the results of some geometrical analyses.

\section{GENUS DIDYMOCYRTIS}

In this study, we focused on images of the genus Didymocyrtis for 2D analysis and a specific specimen of the radiolarian species Didymocyrtis tetrathalamus 
(Haeckel) for 3D analysis. For 3D analysis, we used only data from one specimen because we did not have data of the other species which belong to the same genus.

There are two main reasons for choosing the genus Didymocyrtis: One is based on a paleontological point of view. The fossils of the genus are frequently observed in a wide area of the Earth and for a long period of geological time. This means that the genus is very popular among paleontological researchers. The phylogeny of the genus has been researched in detail (Riedel and Sanfilippo, 1971; Goll, 1972; Kellogg, 1980; Sanfilippo and Riedel, 1980; Suzuki and Aita, 2012; Xiao et al., 2018). There are approximately ten species of Didymocyrtis in the fossil record. The first species of the genus appeared approximately 20.0 Ma. Only one species, D. tetrathalamus, remains presently. Fig. 1 shows a photographic image of a living specimen (Matsuoka, 2017). The other reason is related to geometrical aspects. Its shape is not convex, so its concave shape poses a good challenge. We do not have effective methods for analyzing concave shapes, because we cannot apply the theorems of convex geometry to them.

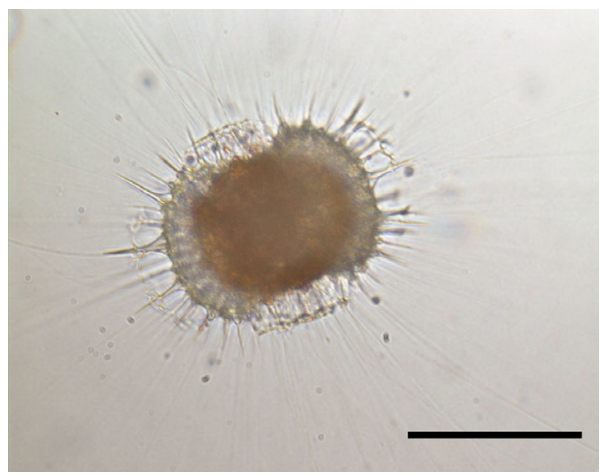

Fig. 1. Photomicrographic image of living Didymocyrtis tetrathalamus. The bar represents $0.1 \mathrm{~mm}$.

The skeleton of Didymocyrtis consists of two parts: an almost spherical shell in the center and a combination of spheroid-like shells which is made up of mainly the outer parts (Anderson et al., 1986; Sugiyama and Anderson, 1998). These two parts are connected by radially extending column-like parts which seem to be located almost in a plane. We refer to the inner spherical part as a medullary shell, the outer concave part as a cortical shell, and the column-like parts as radial bars. There is another part outside of the cortical shell called the extracapsulum (Anderson et al., 1986; Sugiyama and Anderson, 1998); however, we did not consider this part, because it cannot be discussed using geometrical considerations.

We considered the STL data of the skeletal structure of $D$. tetrathalamus acquired using a micro $\mathrm{X}$-ray CT. Fig. 2a shows a snapshot of a 3D image of the data. The acquisition of the data used essentially the same method as Ishida et al. (2015). The width of the specimen is approximately $1 \times 10^{-4} \mathrm{~m}$. Fig. $2 \mathrm{~b}$ shows the same 3D data as Fig. 2a from a different viewpoint which obscures most of the cortical shell in order to display the medullary shell and its connections. As mentioned above, the medullary and the cortical shells are connected by radial bars.

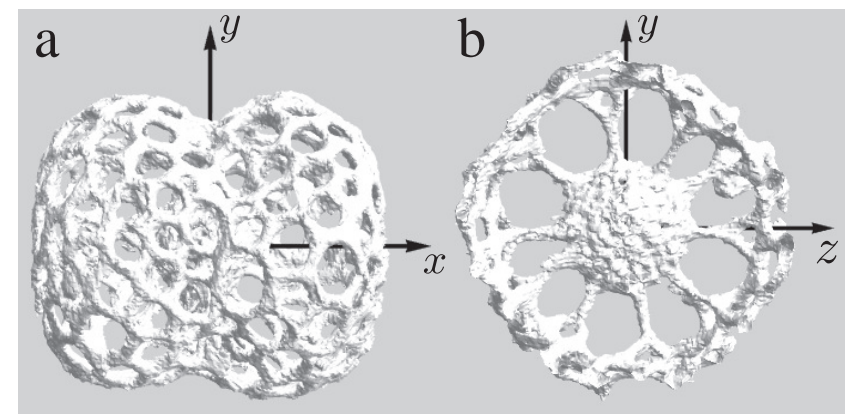

Fig. 2. Image of $3 D$ data with the definition of the coordinates: a) view from $(0,0,10) ; b)$ view from $(10,0,0)$.

\section{ANALYSIS}

We carried out two types of analyses: twodimensional and 3D. The former analysis focused on two-dimensional images and examined the phylogenic sequence of the species. The latter analysis focused on 3D data of a specimen and examined its structure in detail. The 3D data is available on MorphoSource ${ }^{1}$. Throughout the discussion of the analyses, we will present the advantages of introducing a geometrical viewpoint to the research of radiolarian skeletal structures.

We approximated the shape of the cortical shell as two connected spheroids. Fig. 3 illustrates the two spheroids in 3D space which are identical in shape to the cortical shell. We defined the coordinates such that the axis of rotational symmetry is in agreement with the $x$-axis and the $y$ - and $z$-axes lie in the plane of mirror symmetry. As a result, the centers of the spheroids are located on the $x$-axis and their distances

\footnotetext{
${ }^{1}$ https://www.morphosource.org/Detail/Mediaetail/Show/media_id/45653
} 


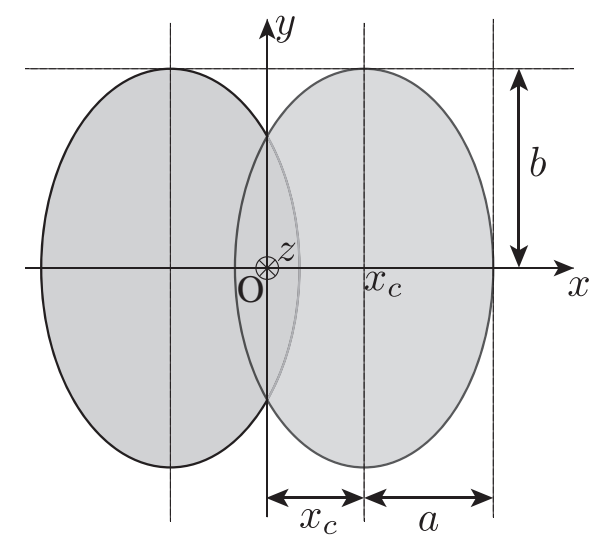

Fig. 3. Ideal shape of a cortical shell and definitions of the coordinates and the parameters.

from the origin are equal. Then the equation of one of our spheroids is

$$
\left(\frac{x-x_{c}}{a}\right)^{2}+\left(\frac{y}{b}\right)^{2}+\left(\frac{z}{b}\right)^{2}=1,
$$

where $x_{c}, a$, and $b$ are positive parameters which characterize its shape. The centers of the two spheroids are described as $\left( \pm x_{c}, 0,0\right)$, where $x_{c}$ denotes the distance of the centers of spheroids from the origin, the ideal center of the shape. The denominators of the second and third terms on the left-hand side, denoted by $b$, are identical because this gives the ideal axisymmetric shape for the approximation of the real cortical shell. We call $a$ and $b$ polar and equatorial radii, respectively.

To focus on the shape of cortical shells, we considered two non-dimensional parameters. The first is referred to as the aspect ratio, defined as $a / b$. The aspect ratio determines the shape of a spheroid. A spheroid is spherical in the case of $a / b=1$ and becomes oblate as $a / b$ decreases. The other parameter is referred to as the distance-diameter ratio, defined as $x_{c} / a$. This ratio is the distance of between the two spheroids normalized by the polar diameter of a spheroid. The two spheroids are more distant from each other as the value increases. By introducing two non-dimensional parameters to our analysis, we can characterize the cortical shells quantitatively.

We considered the two ratios of eight species which belong to the target genus by using the images in Fig. 4.6d of Suzuki and Aita (2012). According to Suzuki and Aita (2012) and Xiao et al. (2018), the phylogenetic evolution of the genus can be summarized by the representative two-dimensional images of the eight species. We obtained measures for $x_{c}, a$, and $b$ from the images and thereby obtained values of the ratios of interest for each image. These ratios were measured by Anderson et al. (1986) only in the case of D. tetrathalamus. Fig. 4 shows the occurrences and lineage of the representative eight species with their species names, photographs, circles approximating their medullary shells, and ellipses approximating their cortical shells. We determined the two ratios based on the ellipses. We could not approximate the cortical shell of $D$. tubaria, because the shape of the shell was far from being connected spheroids.

The values of the ratios changed with the phylogenic evolution of the species as shown in Fig. 5. The figure demonstrates the relation between the appearance of the species and the two ratios. In the case of the aspect ratio, the value decreased with evolution. The results show that the two spheroids corresponding to the cortical shells tend to be oblate, that is, flat. In the case of the distance-diameter ratio, the value increased until $7.8 \mathrm{Ma}$ (appearance of $D$. antepenultima and $D$. penultima), after which it started to decrease. Therefore, the phylogenic change of the cortical shell shape can be summarized as the two spheroids becoming more oblate and more distant from each other until 7.8 Ma, after which they began to move closer together. The evolution followed some kind of strategy and it changed direction around 7.8 Ma. It is notable that the extracapsulum on the outside of the cortical shell, which we did not consider in our analysis, developed extensively from $7.8 \mathrm{Ma}$, as shown in Fig. 4.

We numerically obtained the inertia moment matrix and the principal axes of the 3D data in order to introduce suitable Cartesian coordinates which are consistent with those denoted in Fig. 3. The coordinates were based on the obtained principal axes as shown in Fig. 2. The inertia moment matrix of STL $3 \mathrm{D}$ data $I$ was obtained from the following equation:

$I=\left(\begin{array}{ccc}\sum_{i}\left(\eta_{i}^{2}+\zeta_{i}^{2}\right) A_{i} & \sum_{i} \xi_{i} \eta_{i} A_{i} & \sum_{i} \xi_{i} \zeta_{i} A_{i} \\ \sum_{i} \xi_{i} \eta_{i} A_{i} & \sum_{i}\left(\zeta_{i}^{2}+\xi_{i}^{2}\right) A_{i} & \sum_{i}^{i} \eta_{i} \zeta_{i} A_{i} \\ \sum_{i} \xi_{i} \zeta_{i} A_{i} & \sum_{i} \eta_{i} \zeta_{i} A_{i} & \sum_{i}\left(\xi_{i}^{2}+\eta_{i}^{2}\right) A_{i}\end{array}\right)$,

where $\left(\xi_{i}, \eta_{i}, \zeta_{i}\right)$ and $A_{i}$ respectively denote the center and area of the $i$-th triangle in terms of the original Cartesian $\xi \eta \zeta$-coordinates of the data. The summations are over all triangles. We assumed that the density was uniform and set its value to unity. The principal axes were obtained by the calculation of the eigenvectors of the inertia moment matrix. It is notable that the obtained inertia moment matrix was not correct. This was because STL 3D data record triangles constituting the surfaces of objects. Therefore, the definitions of the coordinates were not based on the volume 


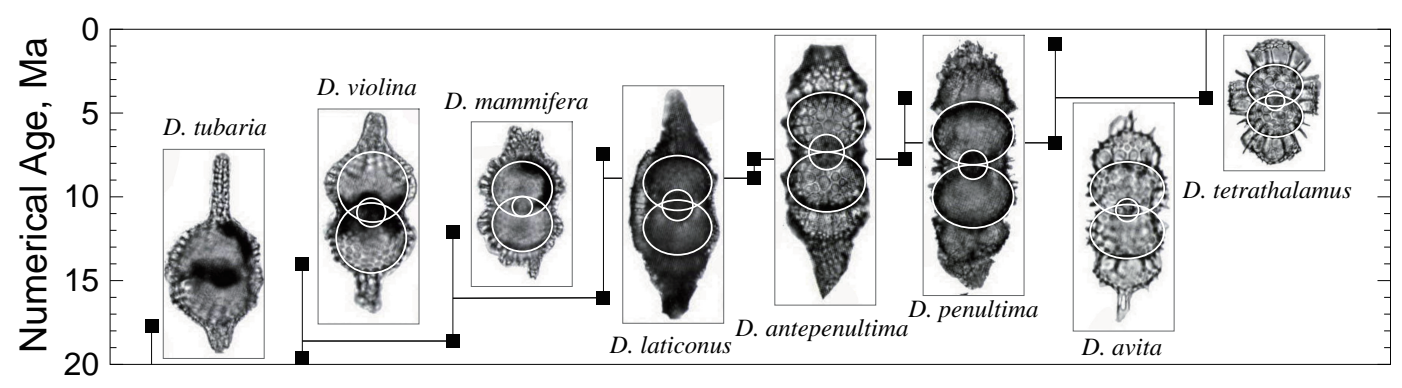

Fig. 4. Phylogenetic evolution of the Didymocyrtis (based on Suzuki and Aita, 2012). Spheroids in the photographs denote the approximated shapes of the cortical shells.

but rather on the centers and the areas of the triangles which the surface consists of. As shown in Fig. 2, the obtained center and the principal axes are adequate to represent the coordinates.

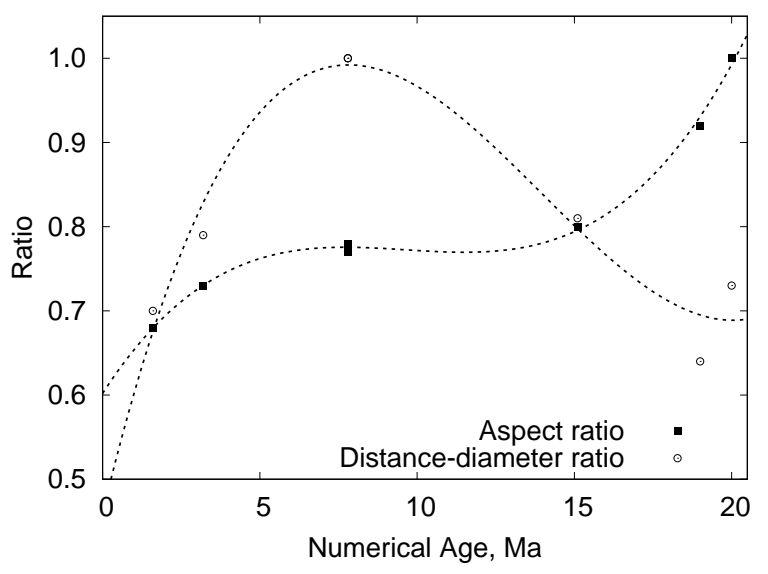

Fig. 5. Sequential changes of the ratios (note that time increases from right to left).

We defined one of the principal axes as the $x$-axis which corresponds to the rotational symmetry axis of the cortical shell and defined the other two principal axes as the $y$ and $z$-axes according to the right-hand system. Thus, we replaced the original axes with the new axes. Fig. 2a shows the projection of the 3D image to the $x y$ plane with the $x$ and $y$-axes denoted by two arrows. We drew spheroids using Eq. 1 with suitable parameters and made them overlap the $3 \mathrm{D}$ image. As a result, we found that the spheroids and the 3D image are approximately the same.

We focused on the number and the distribution of the pores of the cortical shell. Yoshino et al. (2014) proposed a method for determining the pore distribution of a spherical radiolarian skeleton, so we applied this method to our 3D data. We projected the triangles constituting the cortical shell onto a unit sphere in order to apply the method. Note that this means a transformation from a concave structure to a convex one. The number of pores was conserved in this case, but the shapes of the pores changed. This transformation is possible except in the cases of a small aspect ratio and a large distance-diameter ratio when two different points on a spheroid are projected onto the same point on a sphere.

The procedure for finding pores is summarized as follows. Firstly, before the projection onto a spherical surface, we deleted the triangles belonging to the medullary shell and the connecting parts from the 3D image. In other words, we removed all triangles within a given distance from the origin. Secondly, we projected the vertices of the remaining triangles onto a unit sphere and constructed the 3D image as shown in Fig. 6. Finally, we focused only on the vertices of the triangles and approximated them by grid points of a geodesic lattice (Sadouny et al., 1968). Its mesh was dense enough compared to the size of the pores to determine the centers of the pores. Finally, we used the method of Stauffer and Aharony (2014) to count the numbers of domains, the pores, on the lattice.

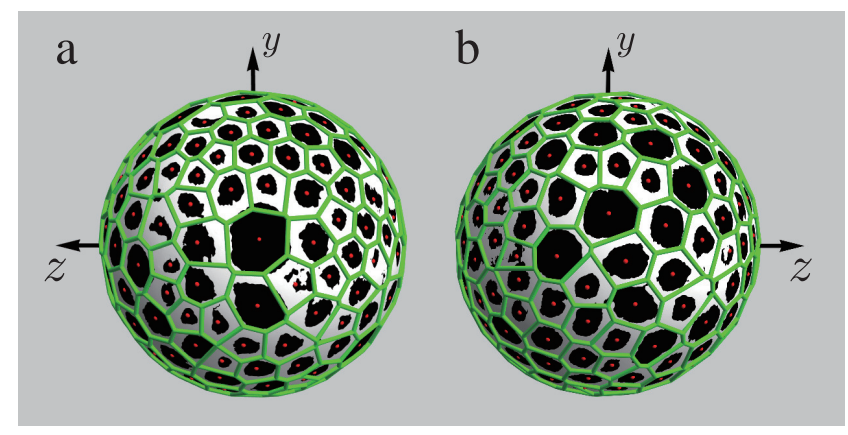

Fig. 6. Projection of cortical shell triangles onto unit sphere: a) view from $(10,0,0) ; b)$ view from $(-10,0,0)$.

The estimated number of pores was 195 and their distribution was neither regular nor symmetric. The resulting images from applying the method of Yoshino et al. (2014) are shown in Fig. 6. No one-to-one correspondence 
or symmetry could be found. We added a black sphere inside the original sphere in order to hide the view seen through the pores. Figs. $6 \mathrm{a}$ and $6 \mathrm{~b}$ show the views along the $x$-axis from the positive and negative viewpoints, respectively. We can observe all of the pores in these two figures because all the pores were projected onto a spherical surface. We added small points at the centers of the detected pores in order to clarify the pores identified. Identification of pores worked well, with a few exceptions. Therefore, the estimated number of pores is an approximate value and the exact value is greater than 196. The rotated and the mirror images of Fig. 6a did not match Fig. 6b. Therefore, we concluded that this skeleton structure did not have any symmetrical properties in terms of pore distribution. This is because symmetry requires a one-to-one correspondence between the pores of the distribution.

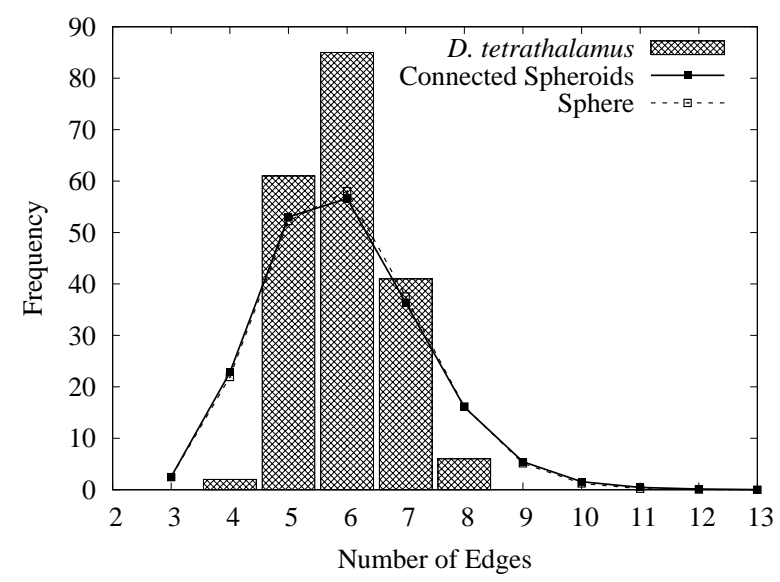

Fig. 7. Frequency distributions of Voronoi polygons. Bar chart: the real specimen. Lines: numerical simulations.

We carried out a spherical Voronoi tessellation using the centers of the pores as the generators in order to consider the distribution of the pores in more detail. We would like to emphasize that the Voronoi tessellation was executed after the translation from the connected spheroids to the unit sphere. The edge numbers of the Voronoi polygons range from four to eight. The bar chart in Fig. 7 shows the frequency distribution of the Voronoi polygons. The mean and standard deviation were 5.938 and 1.205, respectively. The theoretical estimation indicates that if the distribution of generators is uniform on a spherical surface, then most of the polygons are hexagonal except for twelve pentagons. This is because constructing the convex hull from generators uniformly distributed on a sphere results in almost equilateral triangles. Thus, most of their vertices have a degree of six: the dual polygons are hexagons. In our case of 195 generators, the mean and standard deviation of the frequency distribution are 5.938 and 0.240 , respectively. Therefore, the distribution of the pores is not uniform and the deviation from an ideal uniform distribution is caused by the shape of the two connected spheroids and/or the shellforming process.

We obtained the frequency distribution of the edge number of the Voronoi polygons using the 195 generators randomly distributed on the two connected spheroids. We chose the same number as the number of the pores and the same shape as the $3 \mathrm{D}$ data. The method for obtaining the random points used for the generators is summarized in the appendix. After the generation of the random points, we carried out the same procedure as described previously. We obtained 1,000 frequency distributions by using different seeds for the random number generators and report only the mean results. The solid line in Fig. 7 denotes the resulting mean distribution, which differs from that of the original 3D data. In this case, the mean and standard deviation of the frequency distribution were 5.938 and 1.355 , respectively. Although the mean values were the same, the standard deviation of the 3D data was smaller than that of the randomly distributed generators. Therefore, the distribution of the pores was not completely random and some weak effect such as a repulsive one is at work among the pores. In addition, we carried out the same simulation as for the connected spheroids using the 195 generators randomly distributed on a sphere as shown in Fig. 7. The mean and standard deviation of the frequency distribution were 5.938 and 1.312, respectively. The result indicates that the difference of the shape between the connected spheroids and the sphere has no significant effects in the case of the uniformly distributed random generators considered in this study.

We approximated the radial bars using cones. This choice was made because the bars become thicker toward the cortical shell. In order to represent each cone, we assigned suitable values to the positions of apexes, centers of bases, and base radii. We set the apexes to be at the origin, that is, at the center of the 3D data, and express the centers of bases as $\left(r_{\mathrm{b}} \sin \phi_{i}, r_{\mathrm{b}} \cos \phi_{i} \cos \theta_{i}, r_{\mathrm{b}} \cos \phi_{i} \sin \theta_{i}\right)$. The parameter $r_{\mathrm{b}}$ corresponds to the distance from the origin $\left(0<r_{\mathrm{I}}<r_{\mathrm{b}}\right)$ and the angles of the $i$-th cone, $\theta_{i}$ and $\phi_{i}$, determine the direction of the cones, where $r_{\mathrm{I}}$ is the radius of the medullary shell. The $\phi_{i}$ 's denote the deviations of the direction from the $y z$ plane and the $\theta_{i}$ 's are the directions of the cones in the $y z$ plane. We assume that all the cones are identical in shape. Although the outer terminals of the cones split into three branches, we ignored the branches and focused only on the cones.

Fig. 8 shows the resulting images corresponding to three different viewpoints. We displayed the cones in blue and the 3D data as semitransparent. Note that we included a sphere of radius $r_{\mathrm{I}}$ at the origin in order to represent the medullary shell in the image. Furthermore, we added the green cylinders obtained by connecting the centers of the bases. The centers of the bases did not lie in 
the $y z$ plane, as shown in Fig. 8a. The maximum and minimum of the absolute values were 16.0 and 0.57 degrees, respectively. The pattern of deviation was almost perfectly alternating, $(-,-,+,-,+,-,+,-,+,+)$, where each sign denotes the direction of deviation from the $y z$ plane of the cone, in other words, the sign of the $x$-coordinate of the center of the base. The angles between the closest pairs of cones were not equal, as shown in Fig. 8c. The maximum and minimum values were 46.3 degrees and 29.9 degrees, and their mean and standard deviation were 39.5 and 5.24 degrees, respectively. Thus, we concluded that the connections (represented by the cones) were not regular.

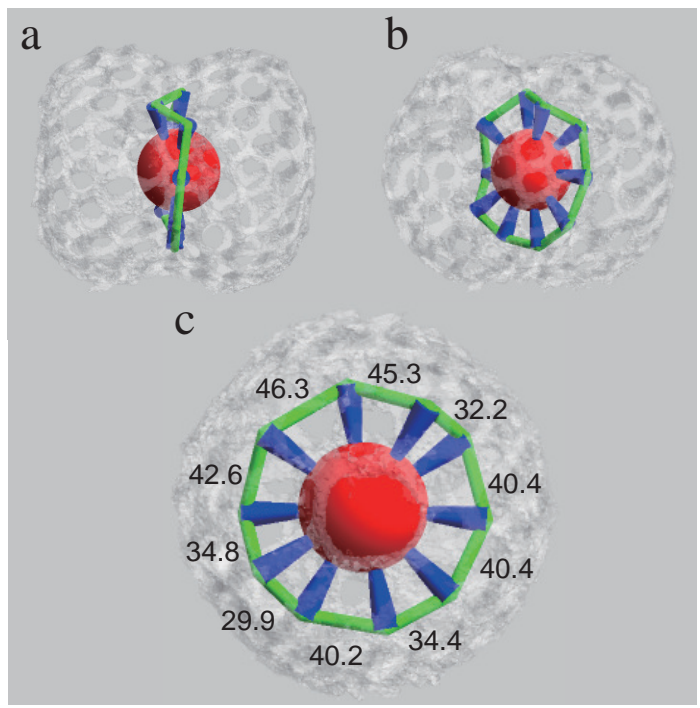

Fig. 8. Representation of the radial bars and the medullary shell with a semitransparent cortical shell: a) view from $(0.63,9.98,0) ; b)$ view from $(7.23,6.85,0)$; c) view from $(10,0,0)$. The numbers are the values of angles between the closest pairs of the cones measured in degrees.

\section{DISCUSSION}

Our approach for introducing geometry revealed new insights into the skeletal structure of D. tetrathalamus and the phylogeny of the genus Didymocyrtis. Therefore, we conclude that the introduction of geometrical considerations provides new information for the research of radiolarians. Although we believe in the usefulness of our approach, some problems remain, as described below.

The first problem is which geometrical properties are useful for describing the phylogenic evolution of Didymocyrtis. We used two ratios in this study; however, it seems likely that there are some other properties which could be used for the description of the evolution. This problem is deeply related to another problem: What is the strategy behind their evolution? This is because the geometrical properties seem to have changed according to evolutionary strategy.

The second problem is the physical role of the cortical shell shape. The shape of the shell reflects its function for the creature. Only the species belonging to this genus have cortical shells that consist of two spheroids. This means that such shapes are an advantage for their survival. In order to consider the advantage, biological or physical treatment of the shape is required.

The last problem is the phylogenic evolutionary strategy of Didymocyrtis. Evolution of a creature can be regarded as an optimization process. Therefore, the changes in the geometrical properties of this genus are also the results of the optimization; however, we could not reveal the objectives of the optimization. We have to consider its evolution from various viewpoints, such as fluid mechanics, biology, and chemistry, in order to clarify the evolutionary strategy.

Our considerations were restricted in the cortical shells and radial bars which develop in the early stage of growth. As mentioned above, we did not discuss the extracapsulum, because it cannot be included within our geometrical considerations. However, this part must be related to the development of the cortical shell as indicated by the identified relation between the $x_{c} / b$ and the development of the extracapsulum. This result indicates that the development of the extracapsulum also influenced the shape of the cortical shell. Therefore, the consideration of the cortical shells can be regarded as an indirect consideration of the extracapsulum, and the geometrical consideration of the extracapsulum is necessary in further research in order to completely clarify the optimization strategy of the skeletal structure.

\section{ACKNOWLEDGEMENTS}

We would like to thank Dr. Noritoshi Suzuki of Tohoku University for permitting us to use the photographs of the eight specimens. We also would like to thank the anonymous referees for their suggestions. This work was supported by JSPS KAKENHI Grant Number JP16K05045.

\section{APPENDIX}

Generation of a random point on the surface of connected oblate spheroids consists of two steps: generation of a random point on a truncated spheroid and translation of the point onto the connected spheroids. First, we generate a random point on the truncated spheroid. A point on the surface of the truncated spheroid $(x, y, z)$ is represented by using two variables $x$ and $\theta$ as 


$$
\begin{aligned}
& x=x, \\
& y=a \sqrt{1-\left(\frac{x}{b}\right)^{2}} \cos \theta, \\
& z=a \sqrt{1-\left(\frac{x}{b}\right)^{2}} \sin \theta,
\end{aligned}
$$

where the ranges of $x$ and $\theta$ are $\left[-x_{c}, b\right]$ and $[0,2 \pi)$, respectively. The range of $x$ is defined in order to avoid the overlapped parts of the two spheroids. A random point on the surface can be obtained by generating two uniform random numbers $x$ and $\theta$ and substituting the values into the equations. Then, we translate the generated random point by $x_{c}$ in the $x$-direction; in other words, we add $x_{c}$ to the $x$ coordinate of the random point. Next, we generate a Boolean random number in order to determine the sign of the $x$ coordinate: the sign is changed if and only if this Boolean random number is zero.

The procedure works well, as shown in Fig. 9, which shows an example of a distribution of 5,000 random points determined using the procedure with the semitransparent spheroids. We chose the same shape as that of the 3D data for the simulation. We used the parameters normalized by $a$ in Fig. 3, so the values of $a$ and $b$ were the unity and aspect ratio, respectively. Thus, $x_{c}$ in the figure is $x_{c} / a$ and the values of the parameters are $a=1.000, b=0.683$, and $x_{c}=0.498$.

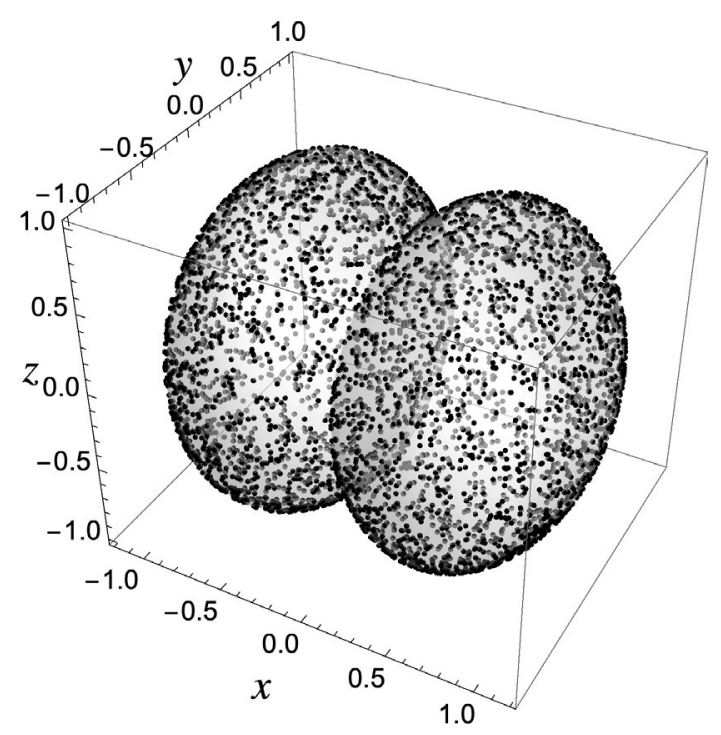

Fig. 9. An example of 5000 random points on connected spheroids.

\section{REFERENCES}

Anderson OR, Hemleben C, Spindler M, Lindsey JL (1986). A comparative analysis of the morphogenesis and morphometric diversity of mature skeletons of living Didymocyrtis tetrathalamus tetrathalamus and Hexalonche amphisiphon. Mar Micropaleontol 11:20315.
Goll RM (1972). Leg 9 Synthesis, Radiolaria. Init Repts DSDP 9:947-1058.

Ishida N, Kishimoto N, Matsuoka A, Kimoto K, Kurihara T, Yoshino T (2015). Three-dimensional imaging of the Jurassic radiolarian Protunuma? ochiensis Matsuoka: an experimental study using high-resolution X-ray micro-computed tomography. Volumina Jurassica 13:77-82.

Kellogg DE (1980). Character Displacement and Phyletic Change in the Evolution of the Radiolarian Subfamily Artiscinae. Micropaleontology 26:196-210.

Matsuoka A (2017). Catalogue of living polycystine radiolarians in surface waters in the East China Sea around Sesoko Island, Okinawa Prefecture, Japan. Science reports of Niigata University. (Geology) 32: 57-90.

Matsuoka A, Yoshino T, Kishimoto N, Ishida N, Kurihara T, Kimoto K, Matsuura S (2012). Exact number of pore frames and their configuration in the Mesozoic radiolarian Pantanellium, An application of X-ray micro-CT and layered manufacturing technology to micropaleontology. Mar Micropaleonto 88-89:36-40.

Riedel WR, Sanfilippo A (1971). Cenozoic Radiolaria from the Western Tropical Pacific, Leg 7. Init Repts DSDP 7:1529-672.

Sadouny R, Arakawa A, Mintz Y (1968). Integration of the Nondivergent Barotropic Vorticity Equation with an Icosahedral-hexagonal Grid for the Sphere. Mon Weather Rev 96:351-6.

Sanfilippo A, Riedel WR (1980). A Revised Generic and Suprageneric Classification of the Artiscins (Radiolaria). J Paleontol 54:1008-11.

Stauffer D, Aharony A (2014). Introduction to percolation theory: revised second edition. Philadelphia: CRC Press, 163-78.

Sugiyama K, Anderson OR (1998). Cytoplasmic organization and symbiotic associations of Didymocyrtis tetrathalamus (Haeckel) (Spumellaria, Radiolaria). Micropaleontology 44:277-89.

Suzuki N, Aita Y (2012). The evolutionary linages of radiolarians. In: Tanimura Y, Tuji A, eds. Microfossils: Their Microscopic World Explored. A Book Series from the National Museum of Nature and Science. 13. Kanagawa: Tokai University Press, 200-5 (in Japanese).

Wagner RC, John R. Jungck JR, Van Loo D (2015). Sub-Micrometer X-ray Tomography of Radiolarians: Computer Modeling and Skeletonization. Microscopy Today 23:18-21.

Xiao Y, Suzuki N, He W (2018). Low-latitudinal standard Permian radiolarian biostratigraphy for multiple purposes with Unitary Association, Graphic Correlation, and Bayesian inference methods. Earth-Sci 
Rev 179:168-206.

Yoshino T, Kishimoto N, Matsuoka A, Ishida N, Kurihara T, Kimoto K (2015). Polyhedron geometry of skeletons of Mesozoic radiolarian Pantanellium. Revue de Micropaléontologie 58:51-6.
Yoshino T, Kishimoto N, Matsuoka A, Ishida N, Kurihara T, Kimoto K (2014). Pores in Spherical Radiolarian Skeletons Directly Determined from ThreeDimensional Data. Forma 29:21-7. 